\title{
Interferometric and spectroscopic monitoring of emission lines
}

\section{Detection of CIRs in hot star winds}

\author{
L. Dessart ${ }^{1}$ and O. Chesneau ${ }^{2}$ \\ 1 N\&S Sterrenkunde Universiteit Utrecht, Princetonplein 5, 3584 CC Utrecht, The Netherlands \\ e-mail: 1.dessart@phys.uu.nl \\ 2 Max-Planck-Institut für Astronomie Königstuhl 17, 69117 Heidelberg, Germany \\ e-mail: chesneau@mpia-hd.mpg.de
}

Received 30 April 2002 / Accepted 31 July 2002

\begin{abstract}
We present a theoretical study of hot star wind variability in the presence of hypothetical large scale wind structures. Contrary to previous investigations that have focused on P-Cygni profiles, we investigate the impact on observable optical and near-infrared emission lines. Our working hypotheses assume that such extended wind structures are formed via a rotationally modulated stellar wind mass loss rate that gives rise to the so-called co-rotating interaction regions and that the modelled wind emissivity suffers from no opacity effect. Within this context, we find that the variability of emission lines traces an unambiguous S-shape in the frequency-time space, i.e. a spiralling pattern with positive and negative accelerations towards the line of sight over one stellar rotation period. Further, we demonstrate how lines forming at different heights can be used to provide dynamical and geometrical constraints on wind structures. Complementary to this spectroscopic approach, we also present theoretical expectations for VLT-AMBER interferometric observations of such a perturbed hot star outflow. For a fixed baseline orientation and length (space-based interferometer), the spectrally dispersed visibility and fringe phase output by the Differential Interferometry (DI) method show strong variable signatures, over a rotation period, of the same nature as those determined from spectroscopy. In the realistic case of both variable length and baseline orientation (ground-based interferometer), the DI method still yields a high detection sensitivity and geometrical characterisation of large scale wind structures.
\end{abstract}

Key words. stars: emission-line, Be - radiative transfer - techniques: interferometry - stars: atmospheres - stars: early type stars: mass-loss

\section{Introduction}

Hot and luminous stars are characterised by large mass loss rates $\left(\dot{M} \sim 10^{-5} M_{\odot} \mathrm{yr}^{-1}\right)$ and terminal velocities $\left(v_{\infty} \sim\right.$ $\left.1000 \mathrm{~km} \mathrm{~s}^{-1}\right)$, resulting from the efficient scattering of continuum star light by optically thick lines of metals (Gayley 1995).

Since the first work of Lucy \& Solomon (1970), Castor et al. (1975, CAK) and Abbott $(1980,1982)$, a successful theory of OB star winds has been developed (Pauldrach et al. 1986), providing reliable determinations for stellar mass loss rates within the single scattering limit. Denser outflows appropriate for the evolved Wolf-Rayet (WR) stars require additional momentum transfer between light and wind material, supposedly achieved by multiple scattering of photons in a denser line opacity distribution (Abbott \& Lucy 1985; Springmann 1994; Gayley \& Owocki 1995). While model atmospheres can reproduce satisfactorily the steady-state properties of these objects - by fitting radiative signatures such as the continuum energy

Send offprint requests to: L. Dessart, e-mail: 1.dessart@phys.uu.nl distribution, emission and P-Cygni line profiles - their steadystate, spherically symmetric and smooth approach offer no explanation for observed time dependent phenomena, indicative of important physical processes.

Indeed, there are numerous indications that hot star winds are in fact quite structured and variable on a range of spatial and temporal scales. Small-scale, stochastic structure is inferred from the black troughs of saturated P-Cygni line profiles, which are understood to arise from the net backscattering of multiple line resonances occurring in a highly non-monotonic velocity field (Lucy 1982, 1984). Embedded wind shocks arising from such velocity variations are moreover thought to give rise to the soft X-ray emission often observed from such hot stars (Lucy \& White 1980; Chlebowski 1989; Feldmeier et al. 1997). This small-scale structure is also identified via the small-amplitude line profile variability (LPV) often detected in high signal-tonoise $(S / N \sim 100-1000)$, optical spectra obtained by groundbased monitoring of recombination emission lines in both OB (Eversberg et al. 1998 on $\zeta$ Puppis) and WR stars (Robert 1992; Lépine \& Moffat 1999). Such small-scale wind structures are 
believed to originate within the wind itself from the unstable nature of line driving (Owocki \& Rybicki 1984, 1985; Owocki et al. 1988; Dessart \& Owocki 2002a).

Large-scale wind structures have also been inferred from the large amplitude variability $(\sim 10 \%)$ in relatively low signalto-noise $(S / N \sim 20)$ IUE spectra, which often show recurring, migrating "Discrete Absorption Components" (DACs) in unsaturated P-Cygni line profiles (Kaper et al. 1997; Prinja et al. 1995). Explaining their longer evolution ( days) than the wind characteristic time-scale ( hours) was theoretically challenging. Following the original idea of Mullan (1984, 1986), Cranmer \& Owocki (1996, CO) studied the impact of some perturbation located at the surface of a rotating star on a radiatively driven wind. Such base perturbations were found to induce the so-called co-rotating interaction regions (CIR) in the stellar outflow, characterised by a velocity plateau and a density compression. Their model was capable of reproducing both the variability level and time-scale observed in P-Cygni absorption troughs (Owocki et al. 1997).

Harries (2000) was the first to investigate the impact of CIRs on emission lines in hot star spectra. Based on a newly developed Monte Carlo code, he modelled the H $\alpha$ LPV for $\zeta$ Puppis (OI) and highlighted the presence of a spiralling variability pattern over a rotation period. While such code permits a 3D modelling of radiative transfer, it requires an analytic and often crude description of the hydrodynamical variables which may affect the accuracy of the computed emergent flux. In this study, we build upon the theoretical success of the CIR model and compute emission line variability based on radiation-hydrodynamics simulations of CIRs. While this permits a more consistent description of the stellar outflow, we restrict our profile simulations to optically thin emission processes, deferring to a future study the possible effects of line opacity. Telescopes can provide standard spectroscopic measurements in the optical and near-infrared, but recently the potential of interferometry as a complementary approach in those spectral bands has been demonstrated vividly. For example, Vakili et al. (1997) used DI to highlight the presence of a (transient) strong asymmetric mass loss in P-Cygni. Also, Vakili et al. (1998) and Berio et al. (1999a) have revealed large scale structures (one-arm oscillations) in Be disks via $\mathrm{H} \alpha$ recombination line monitoring. Hence, large scale wind structures in general, because they induce such obvious wind asymmetry, can be clearly identified by means of interferometry, with such instruments as the near-infrared recombinor AMBER (Petrov et al. 2000) on the Very Large Telescope Interferometer (VLTI). This instrument will indeed permit for the first time the multibaseline temporal monitoring of stellar activity, in the nearinfrared domain with a relatively high spectral resolution.

In this paper, we present the potentials of these two complementary techniques. For this study, we follow the same approach as $\mathrm{CO}$ by generating density-velocity maps of a stellar outflow possessing CIRs (Appendices A and B) and go on to compute two observables: the finely dispersed and spatially unresolved (spectroscopy, Sect. 3) as well as and the coarsely dispersed and spatially resolved (interferometry, Sect. 4) informations on the stellar wind emissivity. In Sect. 5, we present a strategy of observations for VLTI-AMBER and in Sect. 6,

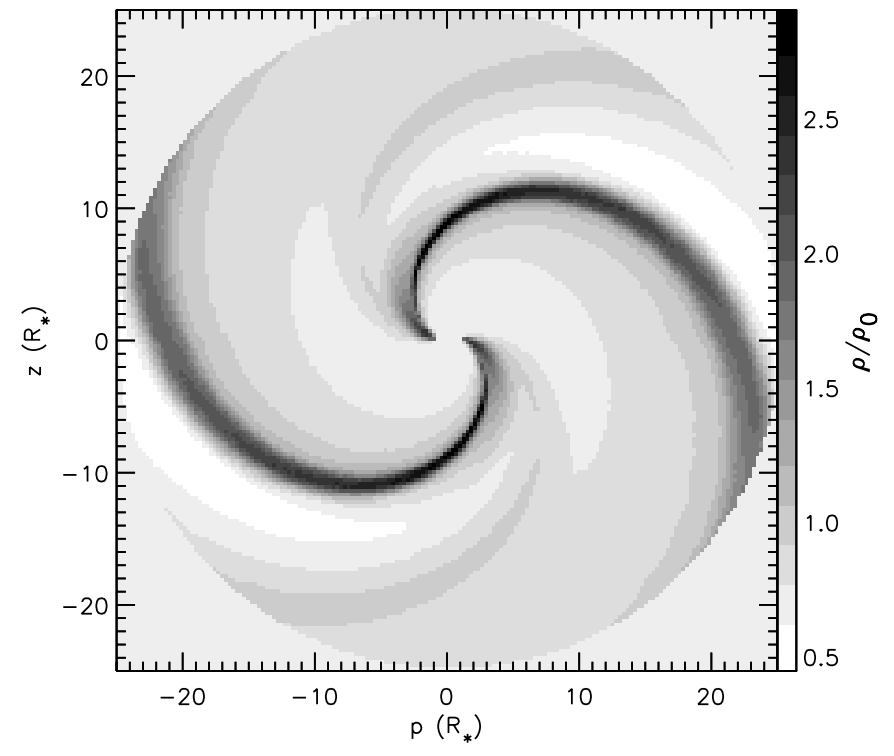

Fig. 1. Equatorial wind density lay-out normalised to the unperturbed case for the basic CIR model used in this study. The details of the model are given in Table 1. Note that these parameters together with the results from the radiation hydrodynamics simulations are identical to those of model 1 in $\mathrm{CO}$. Also, as in $\mathrm{CO}$, we limit the radial extent of the simulation to a maximum radius of $25 R_{\star}$. The velocity field, not shown here, is essentially divergent, i.e. $\boldsymbol{\nabla} \times \boldsymbol{v} \sim 0$ so that the spiral pattern does not represent actual streamlines (see Sect. 2). Selected cross sections of the density and velocity are shown in Fig. 2.

we discuss the limitations of the simulations presented here. Section 7 provides a summary of the work and concluding remarks.

Before dealing with radiative signatures, we discuss in Sect. 2 the key aspects of CIRs in line driven winds.

\section{Description of the wind/CIR structure and dynamics}

Full details on the theoretical properties of CIRs in the context of radiatively driven winds are presented in $\mathrm{CO}$, while here we limit ourselves to the most fundamental of these characteristics and discuss modelling assumptions/techniques in Appendix A.

While the unstable nature of line driving can generate small-scale structures in radiatively driven outflows (mechanism not implemented here), large scale wind structures are believed to be caused by an external perturbation, most likely situated at the base of the wind, which alters the physical conditions in the critical point region where the mass loss rate is set $(\mathrm{CAK})$. As in $\mathrm{CO}$, we postulate here the presence of a surface equatorial perturbation, laterally confined, rotating with the stellar surface, whose effect is to locally enhance the stellar mass loss rate (the bright spot of model 1 in $\mathrm{CO}$ ). This enhancement being transient, the wind becomes overloaded with over-dense and slower material for the subsequent less efficient radiative force. Indeed, once such a "spot" has rotated away, a faster and relatively less dense outflow emerges from the stellar surface and rams into the above material creating a reverse shock with an associated velocity kink/density compression. 
Such a CIR produces a variable mean optical depth evolving on a much longer characteristic time scale than that of the unperturbed wind. And indeed, this wind dynamical structure is believed to be at the origin of the apparent slow acceleration of observed DACs (CO).

Intuitively, one could think that the CIR material follows spiral trajectories in the equatorial plane (Fig. 1). However, the dynamics of this perturbed hot star wind is mostly influenced by the overwhelming radiative force in the radial direction. A spiral pattern arises because different wind portions are affected by the local force enhancement at distinct times, which suffer a time delay as a function of the rotation period and the angle between the two points considered.

We have allowed the outer grid radius to extend outward to $100 R_{\star}$ in some of our simulations, in order to investigate the outer evolution of the CIR (Fig. 2). We find that the CIR density compression vanishes beyond $\sim 30 R_{\star}$, with the concomitant decrease of the absolute density. So, to only include those regions dense enough to contribute to emission profiles, we limit ourselves to $r_{\max }=25 R_{\star}$. The surface perturbation is chosen so that the density contrast of the CIR with the surrounding wind is significant $\left(\left(\rho_{\text {perturbed }} / \rho_{\text {unperturbed }}\right)_{\max } \sim 3\right)$.

Finally, the radiation hydrodynamics simulations used in this work are purely 2D. The computation of emission profiles requires the knowledge of the wind properties in $3 \mathrm{D}$, which are chosen as a hybrid of the calculated perturbed equatorial wind (2D) and an unperturbed polar wind (1D; see CO). This also applies to the spots in that one needs to provide aspect ratios between the latitudinal and azimuthal extents. First, one could just assume that the patch is circular (aspect ratio of unity). However, profile perturbations were more difficult to analyse in that case because the CIR is less confined to the equatorial plane and perturbs a broader range of profile frequencies at any given time. Since we have no knowledge a priori of this aspect ratio, we have chosen a value of $\Delta \phi / \Delta \theta=4$. Also, we calculated the wind equatorial structure over $\phi=[0, \pi]$ with one surface spot and periodic boundary conditions (two spots overall). One surface spot over $2 \pi$ is more costly while having four makes the profile simulations more complex to analyse due to the significant overlap of profile perturbations and nonnegligible dynamical interactions between different CIRs. This paper being merely a theoretical assessment of what we might expect from the presence of CIRs in hot star outflows, a simple set-up is preferred.

We now have the necessary background information to understand the emission profile simulations presented in the following section.

\section{Spectroscopic analysis}

In this section, we present an alternative spectral diagnostics to the widely used P-Cygni profiles in order to improve on our understanding of large scale structures in hot star environments. The P-Cygni profile variability results from the variable wind optical depth between the earth and the stellar disk, mostly affected in practice by the velocity plateau associated with the CIR (CO). Emission lines form instead over spherical shells centred on the star, covering all azimuths. Therefore, they
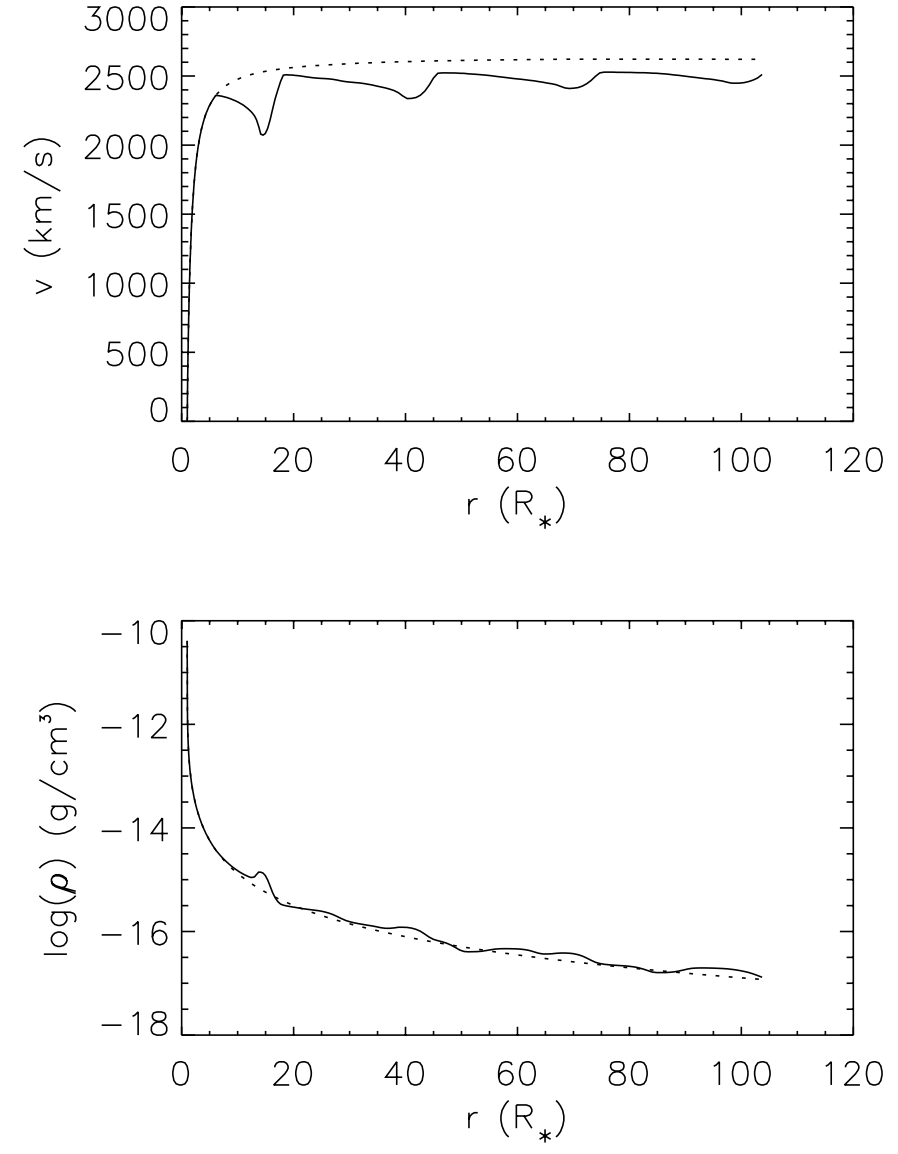

Fig. 2. (top) Wind velocity cross section in the perturbed (solid lines) and unperturbed cases (dotted lines) for a given azimuth and in a simulation with identical parameters to that shown in Fig. 1 but with an extended outer radius. We can clearly identify the velocity plateaus at the origin of P-Cygni profile variability discussed in the study of CO. (bottom) As above but for the wind density. The CIR feature at the origin of the variability of synthetic emission lines is the density compression associated with the CIR.

represent in principle a three dimensional probe of the stellar wind. Their emissivity being proportional to the square of the density, they are an excellent tracer of a density enhancement. Observationally, we will target near-IR lines formed via recombination processes, but our calculations presented here would apply equally well to all density square emission processes.

We assume that this emission process is optically thin, a requirement to be relaxed in our next study. However, much insight can be gained within this context, offering essential information on emissivity diagnostics from CIRs with no photon "loss" after emission. Moreover, this approximation may not be so inadequate for example for WR stars for which numerous optical and near-infrared emission lines show no opacity effect (Dessart \& Owocki 2002b). So, while this assumption breaks down when applied to $\mathrm{H} \alpha \mathrm{LPV}$, it remains applicable to well selected emission lines.

To present the key information contained in emission line variability, we show in Fig. 3 the expected fluctuation of an emission profile in different situations, presented in Table 1. The selected parameter space is chosen to cover the case of a 
Table 1. Model parameters of the profile simulations discussed in Sect. 3. The line emission region (LER) is centred on $r_{\mathrm{c}}$ and extends over $\Delta r$. An optically thin emissivity is assumed. All simulated profiles are presented in Fig. 3. Models A-B (C-D) are chosen to represent a WR $(\mathrm{O})$ star emission line.

\begin{tabular}{lccc}
\hline \hline Model & \multicolumn{2}{c}{ LER } & Inclination \\
& $r_{\mathrm{c}} / R_{\star}$ & $\Delta r / R_{\star}$ & $i(\mathrm{deg})$ \\
\hline $\mathrm{A}$ & 10 & 4 & 20 \\
$\mathrm{~B}$ & 10 & 4 & 70 \\
$\mathrm{C}$ & 3 & 1 & 20 \\
$\mathrm{D}$ & 3 & 1 & 70 \\
\hline
\end{tabular}

WR (OB) emission line formed in the outer wind over extended (restricted) stellar heights. We also investigate the impact of a varying inclination of the system $(i=20,70 \mathrm{deg})$. In Fig. 1, the system is viewed pole-on $(i=0 \mathrm{deg})$ - the equatorial plane and the plane of the sky coincide. The axes of reference are north toward the top of the page, east to the left. Non-zero inclinations correspond to a tilt of the rotation axis westward. In both WR and OB cases, the line forms sufficiently far from the photosphere that the wind has essentially reached its terminal velocity: the profiles are therefore very broad $\left(\sim 2 \times v_{\infty}\right)$. They are also flat-topped due to the assumption of optical thinness.

The central striking feature is that the emissivity enhancement follows a spiralling trajectory in the profile, moving from one side of the profile to the other in a periodic fashion (Harries 2000). Having two axially symmetric CIRs implies the existence of two concomitant spiralling patterns in the profile time sequence. The acceleration of a given sub-peak does not always have the same sign as its line of sight velocity, in contrast to migrating sub-peaks observed in recombination profiles of hot star spectra and associated with small scale structures (Lépine \& Moffat 1999). Indeed, over one rotation period, the density enhancement in the line emission region keeps a constant intrinsic velocity so that the apparent line of sight velocity changes due to the changing line of sight angle $\theta$, which runs over $2 \pi$. The $\cos \theta$ weight appearing in the line of sight velocity runs over $[-1,1]$ and the sub-peaks cover the range $\pm v_{\infty} \cos (i)$. The maximum velocity reached by the CIR profile enhancement therefore gives indirect measure of the inclination (assuming that the CIRs are confined to the equatorial region).

Another aspect is the differing emissivity of a given CIR when moving towards or away from us. This results from the asymmetry of the CIR itself, whose frontal structure is very collimated and rear one more extended and diffuse. This behaviour is stronger in lines forming at smaller heights, the azimuthal configuration of the CIR becoming more forward/backward symmetric away from the photosphere. Note that in the optically thin case treated here, irrespective of the time considered, the total profile emissivity remains constant and it is only the frequency distribution of this emissivity that changes. In all cases, the maximum intensity is reached when the line of sight velocity of a CIR (within the LER) is an extremum, i.e. $\sim \pm v_{\infty} \cos (i)$, or zero. In the former two situations, the profile contribution appears at the same line of sight velocity so
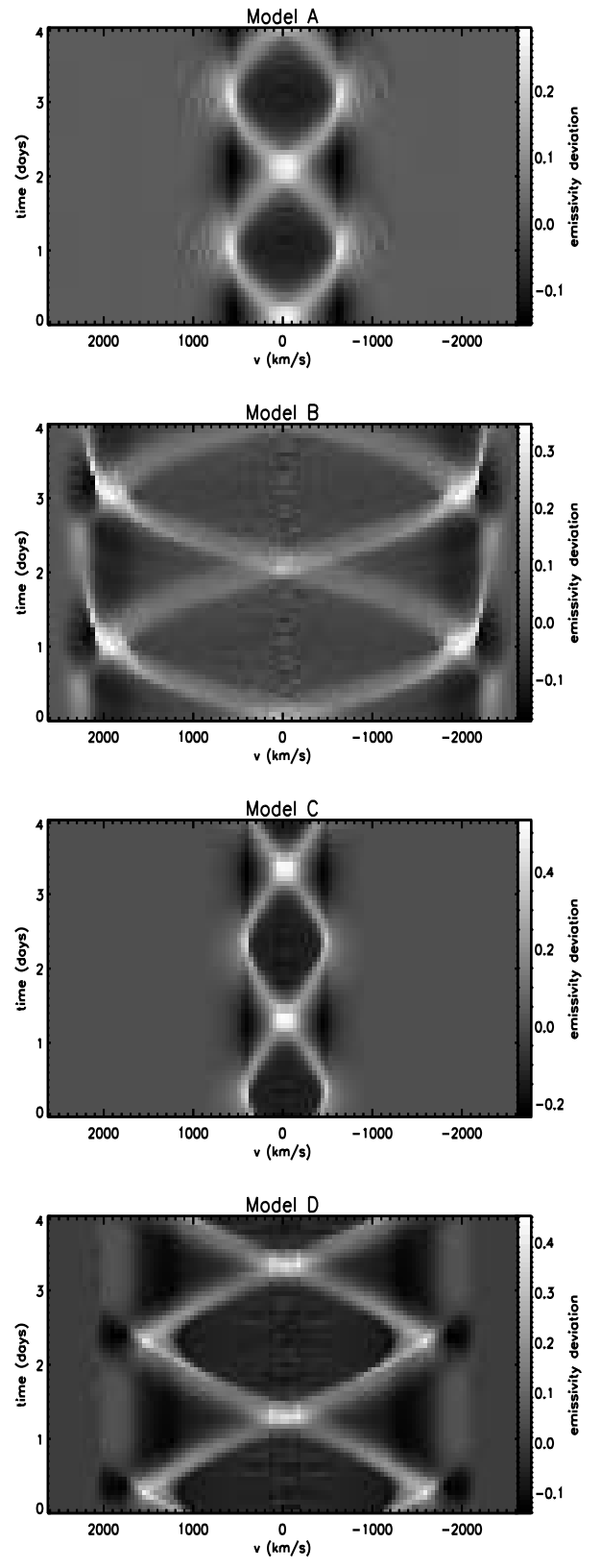

Fig. 3. Intensity plots of the emission deviation from the local time average for models described in Table 1 and in Sect. 3. From top to bottom, we present models A, B, C and D. Not shown here is the average profile, which has simply a square-box shape with a FWHM corresponding to twice the terminal velocity.

that the emissivity due to the density compression falls over a shorter range of profile frequencies (degeneracy), while in the latter the enhancement is caused by the overlapping contributions of the two CIRs at line centre.

The level of fluctuation of the profile emissivity computed here reaches rather high values $(\sim 30 \%)$. Theoretically, the magnitude of this deviation is a measure of the spatial extent as well as the density contrast of the compression. So, quantitatively, this depends entirely on the adopted characteristics of the surface perturbation, for which we have no direct information. In fact, such LPV constitutes an ideal tool for probing the 
photosphere of hot stars, hidden from us because of the optically thick outflow.

Hot star winds are known for their ionization stratification (see Herald et al. 2000 for WR stars). This property can be used to constrain the geometrical extent of putative large scale structures. Indeed, if a large scale structure is present throughout the wind, lines forming at distinct heights will show a variable pattern. Because of the azimuthal extent of that structure, some time delay between the extrema discussed above should become apparent. In our simulations, we clearly identify a phase gap (Fig. 3) for the inner/outer line formation regions treated here. The spiral pattern is observed out of phase (by .7 days for this 4 day period star). The wind structure causing this pattern is therefore extended over $80 \mathrm{deg}$ (azimuthal angle) between these two heights.

Our parameterisation of the ionization stratification is done through height segregation of the emissivity. However, the physics that controls this stratification can heuristically be explained as resulting from the balance between recombination $\left(\propto \frac{1}{v(r) r^{2}}\right)$ and photo-ionization efficiencies $\left(\propto \frac{1}{r^{2}}\right)$. In a spherically symmetric wind, this ratio is only height dependent (in the accelerating wind). In the presence of CIRs, photo-ionization processes are locally superseded by recombination ones. It then appears more adequate to use a density segregation for a given line emissivity (D. Cohen, priv. comm.). Implementing this alternative, we find no significant difference with the former parameterisation.

Have such patterns ever been observed? There has never been any clear indication of a spiralling variability pattern in monitored wind emission lines, even in those stars for which CIRs were strongly suggested from P-Cygni profile variability (see Morel et al. 1998 for EZ CMa). One explanation is that the optical line diagnostics used so far suffer from opacity effects. These alter the escape probability of photons at different line of sight angles for the emitting CIR (Lépine 2001). Similarly, Harries (2000) computed the synthetic LPV of $\mathrm{H} \alpha$ for $\zeta$ Puppis and identifies a spiralling pattern whose intensity (over a rotation period) is higher when it migrates away from line centre. This line forms deep in the wind and therefore suffers significant occultation effects. Also, it is not clear whether the standard wind velocity adopted to describe the CIR affected the synthetic LPV. Using clear flat-topped lines (CIII $5696 \AA$ in WC stars) would offer an ideal alternative to this problem. Another element is that the density compression could be less pronounced in nature than presently calculated, i.e. the surface perturbation is weaker or better quality data is required to reveal a dimmer variability signal. Last, it is to be expected that such surface spots do not necessarily appear at the equator of a rotating star and that the resulting CIR properties deviate significantly from the current ones (see Sect. 6). Numerous surface bright spots located at different latitudes could maybe explain the observed LPV, without resorting to opacity effects.

We have shown that the presence of large scale structures confined to the equatorial plane of a rotating hot star gives unambiguous signatures to emission line variability. We now turn to the investigation of interferometric measurements of such perturbed hot star outflows, as could be obtained with VLTIAMBER.

\section{Interferometric analysis}

The principle of interferometry is to collect light from several telescopes and recombine the different light beams to create an interference pattern (fringes) whose characteristics are function solely of the spatial distribution of the object emission, the spatial separation of the collecting areas (baseline) and the wavelength (Quirrenbach 2001). Here, we use the instrument characteristics of VLTI-AMBER reviewed by Petrov et al. (2000) who highlight a visibility accuracy of about $10^{-2}$ for a spectral resolution $R=1000$ in $J, H, K$ band, and a differential phase rms of the order of 1 degree, i.e. $0.3 \%$, for the $1.8 \mathrm{~m}$ auxiliary telescopes without fringe tracker for a star magnitude of 4-6. Ideally, with considerable amount of observing time and numerous collecting areas, one could map the emissivity on the plane of the sky of a given object in many spectral channels.

However, this cannot be achieved yet by the state-ofthe-art interferometric instrumentation, so that current scientific results are obtained by techniques that require only few baselines and just hours of observations. Combining high angular and spectral resolutions, the method of DI yields (seeingindependent) information on the spatial distribution of the object emissivity and its photocentre on the plane of the sky and as a function of wavelength. This differential measurement is more robust and offers a higher spatial resolution than classical interferometric techniques. For partially resolved objects, DI is ideal to bridge the gap between spectroscopy and reconstructed images provided by full closure interferometry.

In the coming section, we discuss the mathematical content of the interferometric technique. Later in this section, a quantitative assessment of interferometric measurements is provided by radiative transfer calculations based on radiation hydrodynamics simulations of the models of the previous section (see also Appendices A, B).

\subsection{Mathematical description}

For an in-depth description of the method of DI, the reader is referred to Chelli \& Petrov (1995a, 1995b) and Berio et al. (1999b, 2001). Here, we present briefly the fundamental observational quantities, i.e. the visibility or fringe contrast, and the fringe phase related to the position of the object on the plane of the sky. Essentially, the interferometer samples the spatial power spectrum $|\widetilde{O}(\boldsymbol{B} / \lambda)| \mathrm{e}^{i \psi(\boldsymbol{B} / \lambda)}$ of the object brightness $\mathrm{O}(\alpha, \delta)$ at the spatial frequency $\boldsymbol{B} / \lambda(\alpha$ and $\delta$ are the right ascension and the declination of the object). $\boldsymbol{B}$ stands for the interferometric baseline vector projected on the sky and $\lambda$ for the mean wavelength at which the interferometer operates. Thus:

$\mathbf{V}(\boldsymbol{B} / \lambda)=\frac{|\widetilde{O}(\boldsymbol{B} / \lambda)| \mathrm{e}^{i \psi(\boldsymbol{B} / \lambda)}}{|\widetilde{O}(0)|}$

We call visibility the quantity $\frac{|\widetilde{O}(\boldsymbol{B} / \lambda)|}{|\widetilde{O}(0)|}$, and $\psi(\boldsymbol{B} / \lambda)$ the fringe phase.

The fringe phase information $\psi(\boldsymbol{B} / \lambda)$ is lost in principle due to the signal perturbation in the crossing of the atmosphere. It can however be retrieved strictly by the phase closure and 
the dual feeding techniques (Quirrenbach 2001), or relatively by DI. In this latter case, one records the interferometric signal simultaneously at two neighbouring wavelengths, in the continuum acting as a reference $\left(\lambda_{\text {ref }}\right)$ and within the studied emission line $\left(\lambda_{\mathrm{sci}}\right)$. The requirements are that the object are un-resolved by the telescopes taken separately and that the two spectral channels are sufficiently close to avoid wavelength related biases (Berio et al. 2001). Also, $\boldsymbol{B} / \lambda$ is assumed to be the same for both channels, so that the interferometric signal originates solely from differences between the spatial distribution of the target emissivity in the two spectral channels considered.

This technique is free of time-variable noise, for example due to changing atmospheric conditions. Moreover, with this cross-correlation technique, the $S / N$ is proportional to the geometric mean of the flux in each channel. Choosing an extended continuum band provides a high signal, even if the flux/fringe contrast within the science channel is weak. Both relative visibility $\Delta V(\boldsymbol{B} / \lambda)$ and relative fringe phase $\Delta \psi(\boldsymbol{B} / \lambda)$ are obtained physically by reducing dispersed fringes data using a crosscorrelation method between the wavelengths $\lambda_{\text {ref }}$ and $\lambda_{\text {sci }}$. In principle, this differential information is sufficient for our purpose, but an absolute calibration of the reference channel is beneficial.

To a first order, this relative phase reflects a shift of the photocentre of the emission at those two wavelengths and is given by:

$\Delta \psi(\boldsymbol{B} / \lambda)=2 \pi \frac{|B|}{\lambda} \epsilon$

where $\epsilon$ is the projection of the vector distance $(\Delta \alpha, \Delta \delta)=$ $(\alpha, \delta)_{\lambda_{\text {ref }}}-(\alpha, \delta)_{\lambda_{\text {sci }}}$ on the interferometer's baseline. It is theoretically known (Petrov 1988) and practically demonstrated (Vakili et al. 1997, 1998; Berio et al. 1999a) that $\epsilon$ can be much smaller than the angular resolution of the interferometer $\lambda / \boldsymbol{B}$. To ensure the best sensitivity of this technique, we select a large spectral band for the continuum reference.

Now we can discuss the visibility and fringe phase obtained in the context of our CIR simulations.

\subsection{Physical description and simulations}

In practice, interferometry permits the comparison of the emissivity of the system between a selected wavelength band and a continuum reference. Quantitatively, we can simulate the hot star wind emissivity in specific frequency bands covering a given emission line and at chosen positions on the plane of the sky. For a spherically-symmetric radially-expanding smooth wind, we recover the photocentre of isovelocity surfaces, which at all times coincides with the star position. In the presence of large scale structures, asymmetric emissivity contributions are added which shift this photocentre. The frequency band concerned and the magnitude of this shift depend on the characteristics of the perturbed wind at that time, i.e. the line of sight velocity of the CIR density compression, the inclination of the system and line formation region characteristics.

In this study, we wish to address whether the physical properties of CIRs, their number, extent... can be retrieved from the information provided by the interferometric signal. Here, we explore the parameter space of models A to D for which we compute the emissivity maps in eight frequency slices covering the full width of the profile in equal bins $\left(-v_{\infty}\right.$ to $v_{\infty}$, Fig. 4). The star centre coincides with the centre of the annulus/disk represented. The maximum radius is $12 R_{\star}$, and the relative dimensions are maintained between different models and slices. The continuum emissivity contribution from the star is not shown but would in reality be strongly confined spatially and constant in intensity.

In model $\mathrm{A}$, the observer sees the system nearly pole-on, i.e. the equatorial plane and the plane of the sky coincide. For all slices apart from that corresponding to the near mid-plane material (those wind regions which emit at zero line of sight velocity), the emissivity comes entirely from the unperturbed wind. In the slice showing the emitting material with near zero line of sight velocity, we clearly identify a pattern of enhanced emission corresponding to the density compression associated with the CIR. The central slices reveal in fact a very similar pattern to that of Fig. 1, because the observer sees the individual contributions from each region, whose emissivity is a direct function of the density revealed in that figure. We show three more examples (models B, C and D) that vary the height of the emission and the inclination.

As we move towards the edge-on situation, the contrast of emissivities increases so that we finally only see the CIR contribution and hardly any longer the wind. As mentioned in Sect. 3, the total emissivity of the system in the optically thin approach is the same for all inclinations, it is merely the distribution on the plane of the sky and within the slices that changes.

Based on these emissivity maps, we simulate the visibility and the fringe phase as would be observed by VLTI-AMBER (Petrov et al. 2000), the subject of the next section.

\subsection{Signal description}

In our calculation, we consider a baseline of $80 \mathrm{~m}$, and a spectral resolution in $I, J$ and $K$ bands of the order of 1000 , sufficient to resolve large amplitude variations of hot star wind emissivity.

As discussed in Sect. 3, the two sets of models considered so far (A-B and C-D) were chosen to represent line emission in the WR and $\mathrm{O}$ star cases. Because interferometry is sensitive to the spatial distribution of the radiative flux, we need to define the spatial extensions of WR/O star outflows in absolute terms, i.e. the actual dimensions of the continuum and emitting radii. For our model $\mathrm{O}$ star, we choose $\zeta$ Puppis, a nearby bright object $\left(\sim 450 \mathrm{pc}, m_{K}=2.80\right)$, whose apparent angular diameter of $\sim 0.4$ mas (related to $R_{\star}(\tau=2 / 3)$ ) is barely resolved and can be used as a very good interferometric reference. WR stars overall have much smaller hydrostatic radii (about a factor 4) but also possess larger near-IR continuum emission radii (about a factor 4). However, they are typically situated much further away $(\sim 1-2 \mathrm{kpc})$ so that their apparent radii is a factor of a few smaller, i.e. $\sim 0.2$ mas.

In Fig. 5, we present the visibility and the fringe phase calculation for the WR model A (baseline with an east-west orientation). The dotted (solid) line represents expectations for 

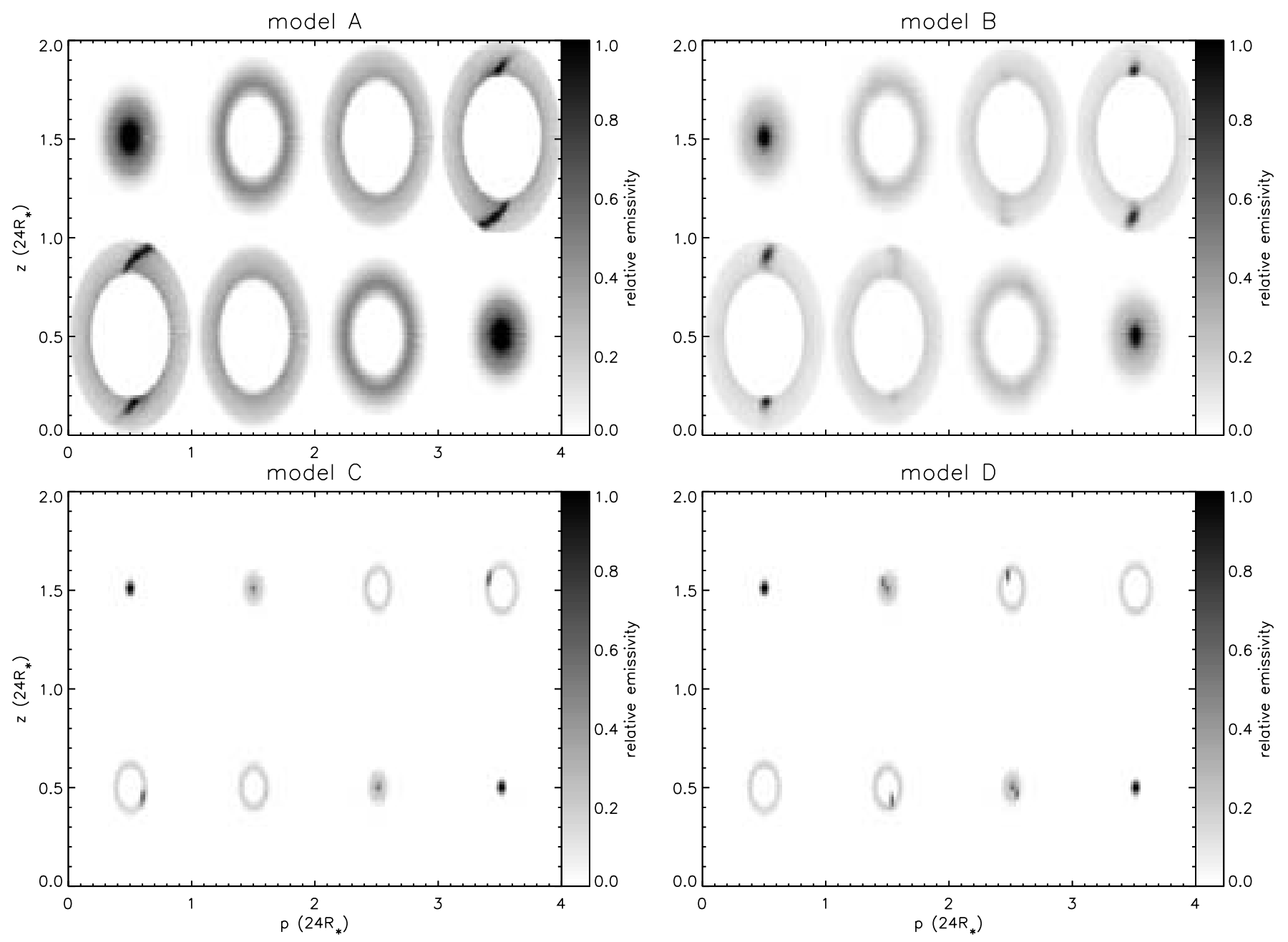

Fig. 4. These four panels describe the spatial distribution on the plane of the sky of the outflow emissivity for models A, B, C and D at time $t=0$ (for comparison with the simulations of Fig. 3). Each panel displays such emissivity in a sequence of eight frequency slices of width $v_{\infty} / 4$, covering the emission profile from $-v_{\infty}$ to $v_{\infty}$. In each of the four panels, the profile is described from blue edge to centre by the slices in the top row, from left to right, and from centre to red edge in the bottom row, again from left to right. Each of these emissivity slices covers in the plane of the sky a square whose side length is $24 R_{\star}$. We specify again the orientation adopted: the zero degree inclination corresponds to the equatorial plane of the system coinciding with the plane of the sky. The axes of reference are north toward the top of the page, east to the left. Non-zero inclinations correspond to a tilt of the rotation axis westward. This further specification is necessary because the system does not have a cylindrical symmetry - these emissivity slices and consequently interferometric measurements (see next section) would be different if the tilt was performed in another direction.

the unperturbed (perturbed) case. Variations in the signal reflect essentially the modulated orientation of the large scale structures with respect to the baseline. The visibility (phase) is maximum (minimum) when the structure lies perpendicular to the baseline. Rotating the baseline by $90 \mathrm{deg}$ reverses those properties (and the grayscales). The continuum visibility (beyond the edges of the line) is nearly unity (unity would be obtained if the star was not just barely but absolutely unresolved). Within the line profile, the visibility of the unperturbed envelope drops to a minimum value of 0.4 . However, its phase is zero due to the exclusive wind emissivity symmetry in those frequency bands - in this situation, a non-zero phase would be a detection of a rotating envelope/photosphere. In the perturbed case, the contribution from the CIR is confined to the central regions of the line ( $\sim 25 \%$ deviation from the unperturbed value).
The phase is non-zero within the profile. Each of the two extrema is related to a given CIR. The "bottom" ("top") CIR shifts the photocentre of the object towards the south (north) at negative (positive) velocities and leads to a negative (positive) phase. The current situation corresponds to a minimum of phase. Later, after rotation, the CIRs are aligned with the baseline and the phase is significantly increased (see temporal evolution of the phase in Fig. 8). Also, in Fig. 4, the spectral resolution is low ( 8 bands over the whole profile) so that the two CIRs appear in one band. In our simulations, to reproduce the spectral resolution of AMBER, we calculated the emissivity maps in 16 frequency channels, which lifted some degeneracy of the CIR presence (spectral resolution of 1000 at $2 \mu \mathrm{m}$ ). We show a similar calculation for model $\mathrm{C}$, i.e. that of an $\mathrm{O}$ star, in order to highlight the signal differences for line diagnostics forming deeper in the wind (Fig. 6). 

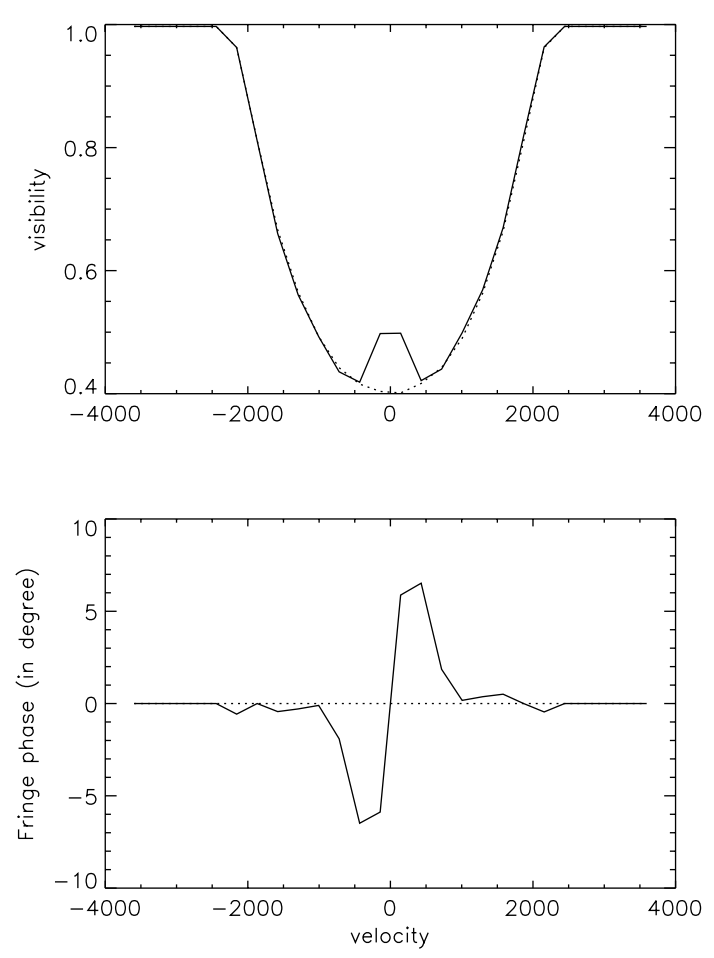

Fig. 5. Perturbed (solid) and unperturbed (dotted) cases for model A. (top): Visibility. (bottom): Fringe phase (in deg). These are the cross sections of Figs. 7 and 8 at the initial time in the series.

These calculations were carried out at a single time and using a unique baseline orientation. In that respect, these simulations do not constitute realistic interferometric measurements because the baseline of a ground-based interferometer evolves with the rotation of the earth. For example, for a two-telescope interferometer, a given observing situation is met only once per night when the hot star emissivity is projected on the same baseline, so that a prohibitive amount of observing time and many different baselines would be required to build the "redundancy" necessary for these simulations. A space interferometer whose orientation can be held fixed can perform easily such measurements. It can be pedagogically instructive to perform a time series for such a fixed interferometer baseline to investigate how the interferometric signal (visibility and fringe phase) evolves due to the changing appearance of CIRs.

In Figs. 7-10, we show these idealised simulations (baseline of $80 \mathrm{~m}$ oriented east-west) and illustrate the close relationship between DI and spectroscopy. The theoretical patterns for spectroscopic and interferometric monitoring follow the same evolution. The phase (visibility) is anti-symmetric (symmetric) about line centre due to our assumption of having two CIRs. Specific times when the CIR contribution falls over several spectral bands reduce the deviation from the un-perturbed case, increasing the difficulty of resolving the emitting CIR. For the WR star case, the signal is almost always detectable: the CIR visibility signal departs from the unperturbed case by up to $5 \%$ over a rotation period, while the phase magnitude reaches values up to $20 \mathrm{deg}$ ( $5 \mathrm{deg}$ at $t=0$, Fig. 5). For the $\mathrm{O}$ star case, the inner location of the wind emissivity makes the visibility and phase signals weaker. However, we find (we show the results
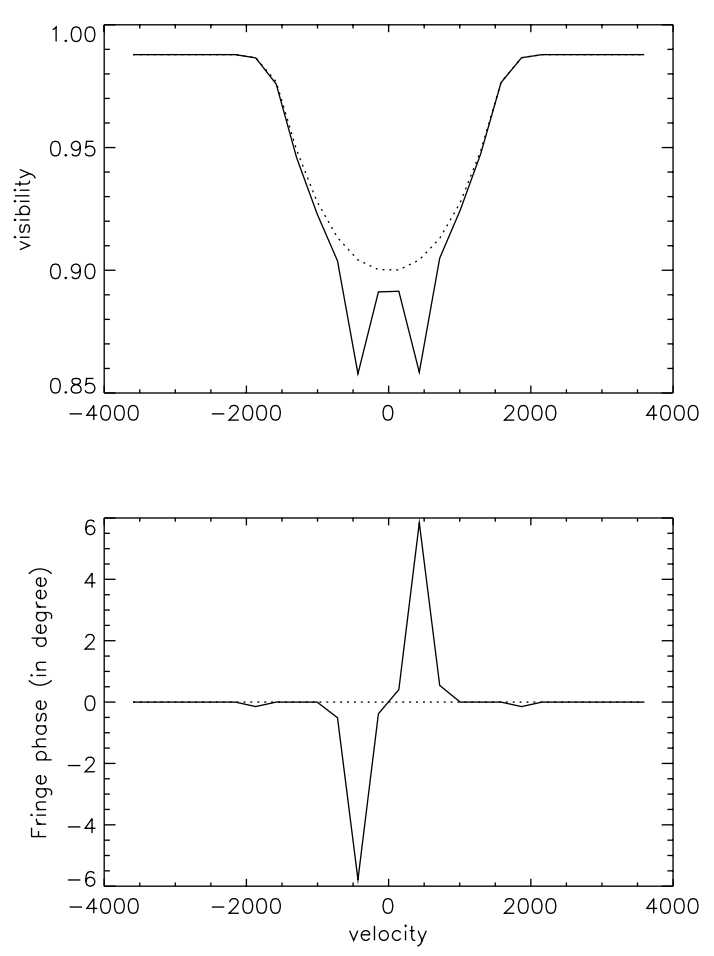

Fig. 6. Perturbed (solid) and unperturbed (dotted) cases for model C. (top): Visibility. (bottom): Fringe phase (in deg).

for models A-B and quote those for model C-D) the visibility signal (max. amplitude of about $3 \%$ ) and the phase signal (max. amplitude of about $5 \mathrm{deg}$ ) are however still detectable in favourable temporal periods (days 0 and 2 for the visibility, 1 and 3 for the phase).

We conclude from this section that the signal from the CIR is at most times strong enough to be resolved, using for our simulations realistic parameters of the AMBER instrument (see beginning of Sect. 4). We have proven that interferometric measurements carried out with a fixed baseline reveal a visibility and fringe phase signal that strongly highlight the presence of CIRs, in a very similar way to what spectroscopy can achieve. In the next section, we move away from the ideal fixed baseline configuration and investigate the results output by a more realistic observational setup.

\section{Feasibility of observations/strategy}

In the previous section, we assumed that the baseline of the interferometer was fixed in time, so that the variability of the signal was a direct reflection of the temporal evolution of the wind emissivity. While informative, this represents a suitable assumption for space-based interferometers. On the ground, interferometers possess baselines whose orientation varies due to the rotation of the earth. Because of that, it is difficult to build measurements under identical telescope/instrument conditions.

One specific constraint in this study is that we target objects whose variability time-scale can be as short as 3 days (e.g. $\zeta$ Puppis and HD 50896), while the rotation of the baseline follows that of the earth (in one day). It is therefore crucial to be able to perform measurements with high enough signal in a 


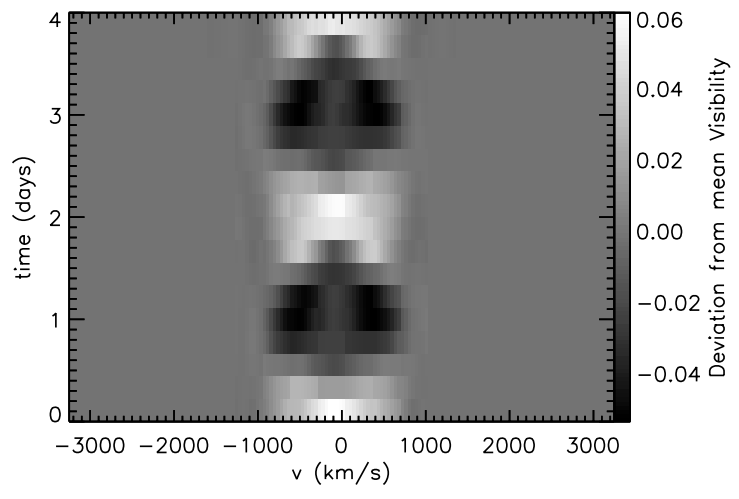

Fig. 7. Temporal evolution of the visibility for model A, compared to the mean over a rotation period.

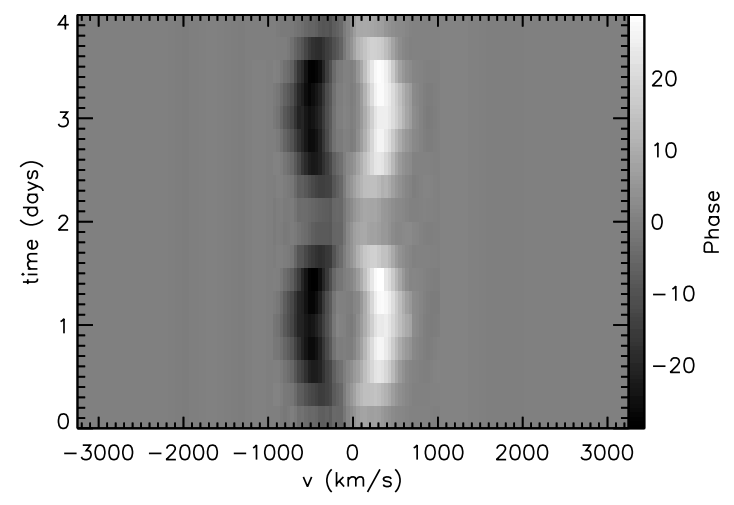

Fig. 8. Temporal evolution of the fringe phase (deg) for model A.

small fraction of the earth rotation time. Flux is not really an issue to achieve a high signal level for our hot luminous program stars. The difficulty is really to reach a sufficient spatial resolution for the fringe sensitivity. This requirement is however met if we use baseline lengths of at least $60 \mathrm{~m}$. To construct the necessary redundancy, a configuration of three equally-spaced telescopes is recommended. This optimises the covering of all directions, and the homogeneity of the projected lengths. It allows also a good determination of the mean visibility signal of the wind. With this setup, we want to reveal the temporal variation of the contrast (visibility) and position (phase) of the fringes in a line compared first to the continuum, and next to subsequent observations. A visibility point with a $S / N \geq 30$ for a spectral resolution $R=1000$ can easily be obtained with an exposure time of one minute and $1.8 \mathrm{~m}$ telescopes for $\zeta$ Puppis. For HD 50896 (WR6), a visibility point is obtained in about $5 \mathrm{~min}$. The calibration of these measurements constitutes the biggest overhead, which we estimate at $30 \mathrm{~min}$. So, one can obtain one visibility point in less than $1 \%$ of these star rotation period. In $6 \mathrm{~h}$, we thus obtain at least a set of 10 independent visibility points per spectral channel corresponding to different baselines and CIR configurations, and a total of about 50 points per CIR period.

At the start, we will search for a non-zero differential fringe phase (line compared to continuum), a non-equivocal evidence of the presence of a significant emissivity asymmetry. Subsequently, if such a deviation is identified as variable in the fringe phase, we will search for it in the visibility signal,

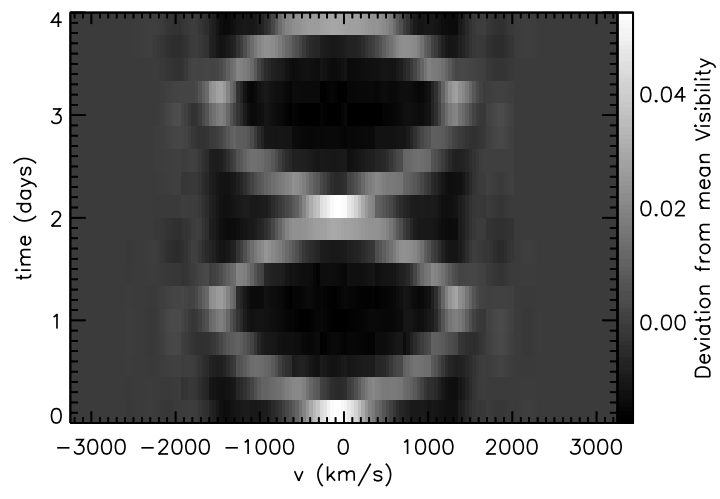

Fig. 9. Temporal evolution of the visibility for model B, compared to the mean over a rotation period.

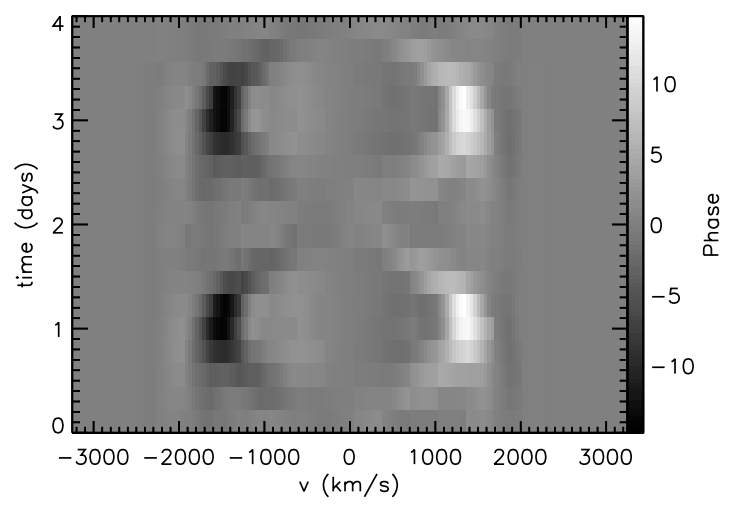

Fig. 10. Temporal evolution of the fringe phase (deg) for model B.

bearing in mind that many observations are needed to build the mean/reference visibility.

One might for example search for a periodic evolution of the fringe phase, which would clearly suggest a periodic emitting configuration. Time series such as shown in Figs. 7-10 cannot be constructed because of the changing baseline. However, we can search for a periodic shift of the emissivity photocentre, as obtained from the fringe phase signal (Eq. (2)). By re-normalising the phase signal by the factor $|B| / \lambda$, we are able to build the periodic shift of the emissivity photocentre. We show in Fig. 11 such a simulation for model A carried out with a three telescope interferometer, covering sixteen consecutive times or half the rotation period of our system (our two-CIR setup makes the signal periodic every half rotation period). A given line-style corresponds to a given time, while positions along a line trace the photocentre position in different spectral bands across the profile. It is clear that in this configuration we can identify a significant shift, periodic in nature. Note that in such near pole-on cases, little or no spectroscopic variability is identified because the temporal fluctuations occur in the mid-plane of the wind as seen by the observer. Interferometry complements well spectroscopy in these situations.

Information about the geometrical structure of the CIRs in the line emission region can be retrieved from Fig. 11. Indeed, we show that at a given time, the photocentre shift follows a line (1D curve) which implies a one-dimensional extent of the 


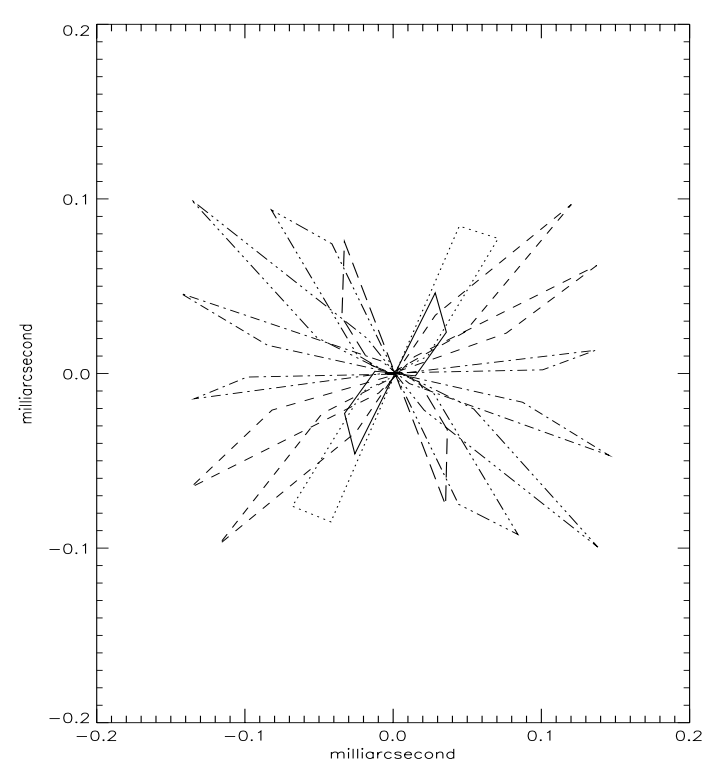

Fig. 11. Shifts of the emissivity photocentre on the plane of the sky over a signal period - half the rotation period - for model A. Different line styles correspond to distinct time (equidistant), while each line traces the photocentre in a given spectral band. Each line follows a unique direction in the sky-plane. Were we to perform the same simulations on a spherically symmetric wind, the emissivity photocentre would coincide with the origin $(0,0)$ at all times and all frequencies.

CIR through the line profile. In the edge-on case (Model B, Fig. 12), a similar calculation reveals the latitudinal extent of the CIR through the 2D migration of the photocentre within the line profile. Note that the line emission region adopted here is too confined to reveal an intrinsic "bending" of the CIRs. Using lines of different ionization at a given time, one could show a photocentre shift in distinct directions, suggesting a different orientation of the CIR, thus providing a similar information to the temporal delay obtained in the spectroscopic variability patterns.

We have demonstrated that we can clearly reveal the presence of large scale structures such as CIRs by means of interferometry. Quantitative information on the spatial structure of the CIR can be obtained especially from the phase information extracted by DI while such information is difficult to extract from spectroscopic measurements.

\section{Limitations}

Our work has made a number of assumptions which can be questionable. First, we have assumed that the emission process is optically thin, mostly for modelling convenience. Indeed, treating line opacity effects is complex and therefore requires a detailed study of its own. We know that such effects might play a role, as demonstrated for example in the phenomenological model of Lépine (2001) for the WR star EZ CMa. Dessart \& Owocki (2002b) discuss line opacity effects in optical emission lines in WR spectra and emphasise that those are generally absent. So, one must wonder whether the observed LPV does not arise from an optically thin line formation process but occurring in a much more complex emitting system, perturbed throughout the wind rather than in the preferred

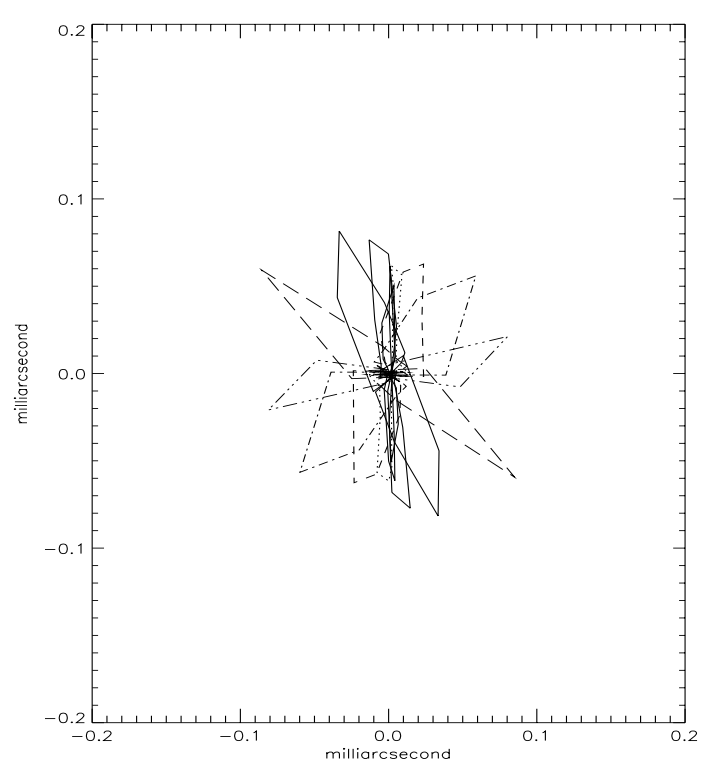

Fig. 12. Same as Fig. 11, for model B. The photocentre shifts are no longer 1D but rather follow a complex path on the plane of the sky, which reflects the latitudinal bending of the CIRs through the line profile.

equatorial plane, with numerous spots, bright and dark, having a spectrum of properties etc. It is clear that optically thick line diagnostics such as $\mathrm{H} \alpha$ (Harries 2000) do not constitute the main focus of the present study. We must wait until a forthcoming study to address the implications for spectroscopic and interferometric measurements of line opacity effects. Together with such investigation, we plan to extend the current radiation hydrodynamics simulations to $3 \mathrm{D}$ as it is not clear whether the complexity of observed LPV arises from opacity effects or from the dynamical and geometrical complexity of the emitting system.

We have studied hot stars with outflows dense enough to produce emission lines. One could also in principle expect continuum wind emission (free-free and bound-free processes), not accounted for here. Since the DI signal is proportional to the difference of intensity distributions between the continuum and the line, an extended continuum will lower the strength of the interferometric signal. Extended emission regions also produce a dilution of the signal (Runacres \& Blomme 1996; Blomme \& Runacres 1997).

We have assumed that the continuum reference is absolutely non-variable. This was motivated by the work of Harries et al. (1998) who highlighted the overwhelming optical variability arising from WR lines compared to the underlying continuum via polarisation studies. But Blomme \& Runacres (1997) suggested the possibility that CIRs could produce continuum eclipse effect when passing through the line of sight. So, it is not too clear whether the continuum might suffer temporal changes due to the presence of large scale structures. Practically, such continuum variability complicates the reduction/analysis but this problem can be solved by using a calibration on a reference star. In that case, using the dual feeding technique, one can retrieve the absolute interferometric signal. 
Finally, observations of hot stars can be problematic when the spectral regions studied are crammed with emission features. Line overlaps merge signals which come from different regions of the wind (hydrogen and helium pickering lines are a typical example in hydrogen-rich hot stars). Hence, selecting adequate lines constitute a crucial step in our work, and in fact in all line variability studies. The associated difficulty is that continuum windows free of emission features are not trivial to find. This problem is however less severe in the near-IR than in the optical for example and can be resolved with sufficient care. The last resort would be to select for the reference band a broad spectral region containing lines and continuum, whose cumulative variability would be minute due to averaging effects.

\section{Conclusions and discussion}

In this paper, we have investigated the impact of large scale wind structures on the temporal evolution of optically thin emission lines. Our working hypothesis was that such large scale structures took the form of CIRs, whose properties were determined from a new implementation within ZEUS-2D of the formalism developed by Cranmer \& Owocki (1996). In this context, the large scale structures are spread in the equatorial plane of the rotating star.

We find that differential interferometric measurements (visibility and fringe phase) can provide a complementary information to that from spectroscopy (emissivity). Indeed, we have found that emission lines forming at intermediate heights $\left(\leq 20 R_{\star}\right)$ show a periodic pattern that spirals the profile line centre, with positive and negative accelerations towards the line of sight over one stellar rotation period. In the ideal case of a fixed baseline (no influence from the earth rotation as for a space-based interferometer), interferometric measurements provide similar results to spectroscopy, i.e. the spiralling pattern is retrieved and its maximum extension in velocity gives the angular tilt of the CIR to the observer. Also, using lines forming at selective heights provides information about the vertical extent of CIRs, while the time shift between patterns gives the lateral extension of such large scale structures. For a more realistic observational setup - the interferometer baseline is no longer fixed - one can however build enough observational redundancy to reveal the presence of large scale structures from photocentre shifts provided by the fringe phase. Interferometry is considerably more spatially sensitive than spectroscopy and is therefore particularly suited to revealing the presence and 3D characteristics of large scale wind structures. Our conclusions assumed a conservative set of instrument characteristics, while larger baselines, 8-m class telescope, adaptive optics will in the future enhance the sensitivity of interferometers even further. Such spatial sensitivity offers a great potential for the study of photospheric structures created by non-radial pulsations or magnetic fields and their distinction from wind structures. Also, the future coupling of AMBER with PRIMA will permit absolute calibration of interferometric signals, solving difficulties with continuum variability.

Theoretically, we wish to improve on the hydrodynamical models used to generate CIRs, by extending to 3D the geometry of our investigation and accounting as well for the angle dependence of the radiation force. A more arbitrary distribution of surface spots perturbing the stellar wind will also be necessary to make our work more general. Also much needed is an assessment of line opacity effects on the computed emissivity.

Observationally, there is a strong need to extend to the nearinfrared region the time monitoring of hot stars, to include lines probing the outer wind. A program with VLT-ISAAC would be an ideal preparation stage for subsequent VLT interferometric measurements, selecting suitable targets for this challenging opening.

Acknowledgements. All hydrodynamical simulations were performed on the basis of the ZEUS-2D code developed at the National Centre for Super-computing Applications (NCSA) at the University of Urbana, Illinois.

\section{Appendix A: Numerical simulations}

The hydrodynamical code used in our work is ZEUS-2D, developed by Stone \& Norman (1992). Magnetism and radiation modules were switched-off during the calculations so that only the hydrodynamical structure of the code was used. ZEUS-2D is a finite difference, Eulerian code, adaptable to various geometries. In the present work, we have slightly modified the code to work in the equatorial plane of the system, in spherical polar rather than cylindrical coordinates. The advection scheme used is the first order Van Leer method. No viscosity is introduced. We assume also that the wind is isothermal, so that the sound speed is everywhere equal to a unique value, that at the surface of the star. The environment of the star is studied from the stellar surface up to $25 R_{\star}$. To reduce the computational time required to run the code, we use a radial increment constant in the logarithm of $3 \%$. We calculate the wind structure to such large radii since we wish to assess directly the level of variability of emission lines that may form very far out.

Table A.1. Model parameters input in the radiation hydrodynamical calculations carried out with ZEUS-2D whose wind temporal evolution is displayed in Fig. 1.

\begin{tabular}{lll}
\hline \hline Quantity & Symbol & Value \\
\hline Mass & $M_{\star} / M_{\odot}$ & 60 \\
Luminosity & $L_{\star} / L_{\odot}$ & $8 \times 10^{5}$ \\
Radius & $R_{\star} / R_{\odot}$ & 19 \\
Equatorial velocity & $v_{\text {eq }}\left(\mathrm{km} \mathrm{s}^{-1}\right)$ & 230 \\
Photospheric density & $\rho_{\star}\left(\mathrm{g} \mathrm{cm}^{-3}\right)$ & $1.2 \times 10^{-10}$ \\
Sound speed & $a\left(\mathrm{~km} \mathrm{~s}^{-1}\right)$ & 24 \\
Ion thermal velocity & $v_{\text {th }}$ & $0.3 \mathrm{a}$ \\
Hydrogen mass fraction & $X$ & 0.71 \\
Helium mass fraction & $Y$ & 0.29 \\
Opacity constant & $k$ & 0.15 \\
CAK power index & $\alpha$ & 0.60 \\
CAK mass loss rate & $\dot{M}_{\mathrm{CAK}}\left(M_{\odot} \mathrm{yr}^{-1}\right)$ & $5 . \times 10^{-6}$ \\
CAK terminal velocity & $v_{\infty}\left(\mathrm{km} \mathrm{s}^{-1}\right)$ & 2600 \\
\hline \multicolumn{2}{c}{ Bright spot characteristics } \\
\hline Angular extent & $\Phi(\mathrm{deg})$ & 20 \\
Perturbation amplitude & $\mathrm{A}$ & 0.5 \\
Number of spots & $\mathrm{N}$ & 2 \\
\hline
\end{tabular}


The uniform azimuthal coverage is 180 deg with 160 points, with periodic boundary conditions in that direction. This explains why we have the simulation of two CIRs, rather than one. We decide to work in an inertial frame, so that the stellar surface rotates like a rigid body while the rest of the flow moves azimuthally according to the prescription of angular momentum conservation. No fiducial force is therefore accounted for in the momentum equation (the Coriolis or centrifugal terms). We implement the radiation force and the boundary conditions as in $\mathrm{CO}$, where full details on this type of simulations are given. In particular, we make no allowance for the radiative instability mechanism. We warn the reader that only the radial component of the radiation force is included. The presence of 1(n) bright spot(s) leads to a local enhancement of the line driving, whose angular and radial dependence are implemented as in $\mathrm{CO}$ (their function $\gamma(r)$ together with the gaussian shape of the azimuthal amplitude modulation). The line force modulation due to the presence of a spot is confined to nearby azimuth via a gaussian multiplicative factor.

Following their nomenclature, we give in Table A.1 the characteristics of the bright spots used. Also in this table are the stellar characteristics used in the hydrodynamical simulations, representative of $\zeta$ Puppis (Kudritzki et al. 1983), while it is a trivial matter to extend our work to other luminous stars whose winds are generated by radiation pressure in a large ensemble of optically thick and thin lines (CAK).

We find both a qualitative and a quantitative agreement with the simulations carried out by $\mathrm{CO}$, and provide one snapshot for the case of two bright spots in Fig. 1.

\section{Appendix B: Recombination profile simulations}

The method presented here overlaps considerably with that of DO. It also makes use of the approximations discussed in CO. The inputs to the profile simulator are generated by ZEUS-2D (see Sect. 2 and Appendix A). This provides the equatorial wind structure, while the 3D outflow has to be characterised in order to calculate an emission line profile. As in $\mathrm{CO}$, at any wind point $(r, \theta, \phi)$, the density/velocity is defined as a hybrid between the calculated equatorial value at $(r, \phi)$ and and its assumed un-perturbed value at $r$ obtained from a standard 1D CAK wind with identical parameters (those for $\zeta$ Puppis without any base perturbation).

The present wind properties show an angle correlation due to the rotation of the system, the periodic perturbation of the wind by the spot. Hence, it is quite different from the entirely radial treatment of the patch approach described in DO. Still, the data is organised as star centre cones, so that for each $(\theta, \phi)$, we have a number of "slabs" running over all radii whose angular extent is set by the resolution used in the radiation hydrodynamics code. Further, we can treat the profile contributions as in the patch method, i.e. as arising from independent slabs bounded in radius, azimuth and latitude. The profile frequency where the contribution is made depends on the projected velocity along the line of sight of each slab, spanning a frequency range set by the maximum and minimum line of sight angles of that cell, as seen by the viewer.
While the patch approach described in DO assumed no symmetry of any kind, here the strong equatorial patterns differ greatly from the properties at other latitudes so that we expect to observe a very different recombination profile depending on the inclination of the system and the equatorial structure as a function of azimuth.

The line of sight angle of a given cell will now determine the projected velocity of the velocity vector of that cell. Suppose the spherical coordinate of the viewer are $\left(\theta_{\mathrm{v}}, \phi_{\mathrm{v}}\right)$; the cell $(\theta, \phi)$. The angle between the viewer and the cell as seen at the star centre is:

$$
\begin{aligned}
\alpha= & \cos ^{-1}\left(\sin \theta \cos \phi \sin \theta_{\mathrm{v}} \cos \phi_{\mathrm{v}}\right. \\
& \left.+\sin \theta \sin \phi \sin \theta_{\mathrm{v}} \sin \phi_{\mathrm{v}}+\cos \theta \cos \theta_{\mathrm{v}}\right)
\end{aligned}
$$

since the unit vectors of the viewer and the cell are respectively (in star centred coordinates):

$$
\begin{aligned}
& \boldsymbol{n}_{\mathrm{v}}=\sin \theta_{\mathrm{v}} \cos \phi_{\mathrm{v}} \boldsymbol{x}+\sin \theta_{\mathrm{v}} \sin \phi_{\mathrm{v}} \boldsymbol{y}+\cos \theta_{\mathrm{v}} \boldsymbol{z} \\
& \boldsymbol{n}_{\mathrm{c}}=\sin \theta \cos \phi \boldsymbol{x}+\sin \theta \sin \phi \boldsymbol{y}+\cos \theta \boldsymbol{z} .
\end{aligned}
$$

The projected velocity of the cell along the direction to the viewer is simply $v_{\mathrm{LOS}, \alpha}=\boldsymbol{v} \cdot \boldsymbol{n}_{\mathrm{v}}$. We assume that the frequency span results from the geometrical extent of the cell $(\Delta \phi)$ and given by

$v_{\mathrm{LOS}, \alpha \pm \Delta \phi / 2}=v_{\mathrm{LOS}, \alpha} \frac{\cos (\alpha \pm \Delta \phi / 2)}{\cos \alpha}$.

The computation of the recombination line emissivity is done as in DO (Sect. 3.2). To construct the synthetic recombination profile, we run cover all $\theta$ angles between $[0, \pi]$, with the $m(\theta)$ individual "patches", and run over all radii. This is identical to the patch method, except that here, the profile contribution may appear at differing frequency positions depending on the inclination.

To calculate the emergent profile from a 3D wind geometrically treated with those patches, we sum up the contributions from all patches. For each of the $m\left(\theta_{i}\right)$ in annulus $\theta_{i}$ and at each radius $r$, the differential contribution to the emission is given by

$\mathrm{d} L_{x}\left(r, \theta_{i}\right) \propto \begin{cases}\frac{2 \pi}{m(\theta)} \frac{v_{\infty}}{v(r)} r^{2} \rho^{2}(r) \mathrm{d} r & \text { for } x_{\min , i}<x<x_{\max , i} \\ 0 . & \text { otherwise }\end{cases}$

where the minimum and maximum profile frequency of the contributions are given by $x_{\min , i}=v_{\mathrm{LOS}, \alpha+\Delta \phi 2 /} / v_{\infty}$ and $x_{\max , i}=$ $v_{\mathrm{LOS}, \alpha-\Delta \phi / 2} / v_{\infty}$. As in DO, this method is tested against the prediction of the so-called shell approach assuming spherical symmetry. We find that for all inclinations, the departure between the patch and shell methods in this ideal case lead to no more than a $1 \%$ difference, all profile frequencies considered.

\section{References}

Abbott, D. C. 1980, ApJ, 242, 1183

Abbott, D. C. 1982, ApJ, 259, 282

Abbott, D. C., \& Lucy, L. B. 1985, ApJ, 288, 679

Berio, P., Stee, P., Vakili, F., et al. 1999a, A\&A, 345, 203

Berio, P., Mourard, D., Bonneau, D., et al. 1999b, JOSA, 16, 872

Berio, P., Mourard, D., Pierron, M., \& Chelli, A. 2001, JOSA, 18, 614

Blomme, R., \& Runacres, M. C. 1997, A\&A, 323, 886 
Castor, J. I., Abbott, D. C., \& Klein, R. I. 1975, ApJ, 195, 157 (CAK) Chlebowski, T. 1989, ApJ, 342, 1091

Chelli, A., \& Petrov, R. G. 1995a, A\&AS, 109, 389

Chelli, A., \& Petrov, R. G. 1995b, A\&AS, 109, 401

Cranmer, S. R., \& Owocki, S. P. 1996, ApJ, 462, 469

Dessart, L., \& Owocki, S. P. 2002a, A\&A, 383, 1113

Dessart, L., \& Owocki, S. P. 2002b, A\&A, in preparation

Eversberg, T., Lépine, S., \& Moffat, A. F. J. 1998, ApJ, 494, 799

Feldmeier, A., Puls, J., \& Pauldrach, A. 1997, A\&A, 322, 878

Gayley, K. G. 1995, ApJ, 454, 410

Gayley, K. G., \& Owocki, S. P. 1995, ApJ, 446, 801

Harries, T. J. 2000, MNRAS, 315, 722

Harries, T. J., Hillier, D. J., \& Howarth, I. D. 1998, MNRAS, 296, 1072

Herald, J. E., Schulte-Ladbeck, R. E., Eenens, P. R. J., \& Morris, P. 2000, ApJS, 126, 469

Kaper, L., Henrichs, H. F., Fullerton, A. W., et al. 1997, A\&A, 327, 281

Kudritzki, R. P., Simon, K. P., \& Hamann, W. R. 1983, A\&A, 118, 245

Lépine, S., \& Moffat, A. F. J. 1999, ApJ, 514, 909

Lépine, S. 2001, priv. comm.

Lucy, L. B., \& Solomon, P. 1970, ApJ, 159, 879

Lucy, L. B. 1982, ApJ, 255, 278

Lucy, L. B. 1984, ApJ, 274, 372
Lucy, L. B., \& White, R. 1980, ApJ, 241, 300

Morel, T., St-Louis, N., Moffat, A. F. J., et al. 1998, ApJ, 498, 413

Mullan, D. J. 1984, ApJ, 283, 303

Mullan, D. J. 1986, A\&A, 165, 157

Owocki, S. P., \& Rybicki, G. B. 1984, ApJ, 284, 337

Owocki, S. P., \& Rybicki, G. B. 1985, ApJ, 299, 265

Owocki, S. P., Castor, J. I., \& Rybicki, G. B. 1988, ApJ, 335, 914 (OCR)

Owocki, S. P., Cranmer, S. R., \& Fullerton, A. W. 1997, ApJ, 453, 37 Pauldrach, A., Puls, J., \& Kudritzki, R. P. 1986, A\&A, 164, 86

Petrov, R. 1988, in High-Resolution Imaging by Interferometry, ESO Conf., March 1988, Garching, ed. F. Merkle (Garching, Germany), 235

Petrov, R., Malbet, F., Richichi, A., Hoffman, K. H., Mourard, D., et al. 2000, SPIE, 4006, 68

Prinja, R. K., Massa, D., \& Fullerton, A. W. 1995, ApJ, 452, 61

Quirrenbach, A. 2001, ARA\&A, 39, 353

Robert, C. 1992, Ph.D. Thesis, Univ. Montréal

Runacres, M. C., \& Blomme, R. 1996, A\&A, 309, 544

Stone, J. M., \& Norman, M. J. 1992, ApJ, 80, 753

Springmann, U. 1994, A\&A, 289, 505

Vakili, F., Mourard, D., Bonneau, D., Morand, F., \& Stee, P. 1997, A\&A, 323, 183

Vakili, F., Mourard, D., Stee, P., et al. 1998, A\&A, 335, 261 